\title{
Generalized golden ratios and associated Pell sequences
}

\author{
A. G. Shannon ${ }^{1}$ and J. V. Leyendekkers ${ }^{2}$ \\ ${ }^{1}$ Warrane College, The University of New South Wales, NSW 2033, Australia \\ e-mail: tshannon38@gmail.com \\ ${ }^{2}$ Faculty of Science, The University of Sydney, NSW 2006, Australia \\ e-mail: jeanvaldek@gmail.com
}

Received: 16 May 2018

Accepted: 30 August 2018

\begin{abstract}
This paper considers generalizations of the golden ratio based on an extension of the Pell recurrence relation. These include related partial difference equations. It develops generalized Pell and Companion-Pell numbers and shows how they can yield elegant generalizations of Fibonacci and Lucas identities. This sheds light on the format of the original identities, such as the Simson formula, to distinguish what is significant and substantial from what is incidental or accidental.

Keywords: Golden ratio, Fibonacci numbers, Lucas numbers, Pell numbers, Companion-Pell numbers, Simson's identity, Binet formula, Recurrence relations, Difference equations, Pythagorean triples.
\end{abstract}

2010 Mathematics Subject Classification: 11B39.

\section{Introduction}

Each of the equations:

$$
\begin{array}{r}
x^{2}-x-1=0 \\
x^{3}-2 x^{2}+1=0 \\
2 x^{4}-5 x^{3}+4 x+1=0
\end{array}
$$

has two of its roots $\frac{1}{2}(1 \pm \sqrt{5})$, because each polynomial has $x^{2}-x-1$ as factor. The positive root of (1.1) is numerically equal to $\varphi$, the Golden Ratio, known since the time of Euclid [1]. Simply, $\varphi$ is the ratio of two line segments (major/minor) and simultaneously the ratio of the total line 
length over the major line segment (that is, both ratios are equal). For notational convenience, we let $1-\varphi=\psi$.

Furthermore, $\varphi$ is essential to a multitude of natural patterns, from sunflower florets to the shapes of galaxies and has been studied extensively over the centuries. In 1509 Pacioli published a three volume treatise on the "Divine Proportion", that is $\varphi$. The ratio has been used in the novel Goldpoint Geometry [2]. We consider here some other properties of $\varphi$ to generalize some wellknown second order recursive sequences.

\section{The Golden Ratio and Fibonacci numbers}

The powers of $\varphi$ are related to the elements of the Fibonacci sequence, $\left\{F_{n}\right\}$ [3]:

$$
\begin{aligned}
& \varphi^{n}=F_{n} \varphi+F_{n-1}, \\
& \psi^{n}=F_{n+1}-F_{n} \varphi .
\end{aligned}
$$

These power relations were known to Euler (1707-1783) and de Moivre (1667-1754) and rediscovered by Binet (1786-1856), one of the discoverers of the formula for the general term of the Fibonacci sequence [4]:

$$
F_{n}=\frac{\varphi^{n}-\psi^{n}}{\sqrt{5}}
$$

We note the corresponding formula for the general term of the Lucas sequence:

$$
L_{n}=\varphi^{n}+\psi^{n}
$$

so that

$$
L_{n}=F_{n-1}+F_{n+1}
$$

Thus,

$$
\frac{F_{n+1}}{F_{n}}=\frac{\varphi^{n+1}-\psi^{n+1}}{\varphi^{n}-\psi^{n}}
$$

so that

$$
\lim _{n \rightarrow \infty} \frac{F_{n+1}}{F_{n}}=\varphi
$$

which can be used in calculating Fibonacci numbers for 'large' $n$, even though $\varphi$ is irrational and $\frac{F_{n+1}}{F_{n}}$ is rational. Nevertheless, the decimal patterns for the latter can have a very long interval before repeating; for example, where the last digits of $F_{n}, n>3$, have a periodicity of $15 \times 10^{m-1}$ where $m$ is the number of end digits Thus, for $m=3$ the periodicity of the three end digits is 1500 . This can lead to relatively large decimal repeat patterns for the golden ratio:

$$
\frac{F_{18}}{F_{17}}=\frac{2584}{1597}=1.61803381340001252340815278647463994990607388854101440003757044583 \ldots
$$

Stakhov [5] used Table 1 below to prove various results for $\varphi^{n} \pm \psi^{n}$. 


\begin{tabular}{|c|c|c|c|c|c|c|c|c|}
\hline $\boldsymbol{n}$ & $\mathbf{0}$ & $\mathbf{1}$ & $\mathbf{2}$ & $\mathbf{3}$ & $\mathbf{4}$ & $\mathbf{5}$ & $\mathbf{6}$ & $\mathbf{7}$ \\
\hline$\varphi^{n}$ & $\frac{-2+0 \sqrt{5}}{2}$ & $\frac{1+\sqrt{5}}{2}$ & $\frac{3+\sqrt{5}}{2}$ & $\frac{4+2 \sqrt{5}}{2}$ & $\frac{7+3 \sqrt{5}}{2}$ & $\frac{11+5 \sqrt{5}}{2}$ & $\frac{18+8 \sqrt{5}}{2}$ & $\frac{29+13 \sqrt{5}}{2}$ \\
\hline$\psi^{n}$ & $\frac{-2+0 \sqrt{5}}{2}$ & $\frac{-1+\sqrt{5}}{2}$ & $\frac{3-\sqrt{5}}{2}$ & $\frac{-4+2 \sqrt{5}}{2}$ & $\frac{-7+3 \sqrt{5}}{2}$ & $\frac{11-5 \sqrt{5}}{2}$ & $\frac{-18+8 \sqrt{5}}{2}$ & $\frac{29-13 \sqrt{5}}{2}$ \\
\hline
\end{tabular}

Table 1. Stakhov's powers of the golden ratio

The appearance of the Lucas and Fibonacci numbers in the numerators is no accident. Stakhov's context was a foundation for computer arithmetic [cf. 6], but his Table 1 also suggests extending, for instance, $\frac{Q_{1}+P_{1} \sqrt{8}}{2}$ in which $\left\{P_{n}\right\}$ and $\left\{Q_{n}\right\}$ are the classical Pell $\{1,2,5,12, \ldots\}$ and Companion-Pell $\{2,2,6,14,34, \ldots\}$ sequences respectively, which we shall attempt in the next section. Here we set

$$
\alpha_{k}^{n}=\frac{L_{k, n}+F_{k, n-1} \sqrt{k^{2}+4}}{2}
$$

and

$$
\beta_{k}^{n}=\frac{L_{k, n}-F_{k, n-1} \sqrt{k^{2}+4}}{2}
$$

in a manner analogous to that of [7]. $k \in Z$ here is associated with generalized Pell $\left\{P_{k, n}\right\}$ and Companion-Pell sequences $\left\{\mathrm{Q}_{k, n}\right\}$ [8] which satisfy the linear homogeneous second order recurrence relation:

$$
P_{k, n+1}=k P_{k, n}+P_{k, n-1}, n \geq 1,
$$

with initial conditions (for notational convenience) $P_{k, 0}=1, P_{k, 1}=k, Q_{k, 0}=2, Q_{k, 1}=k$, to include traditional Fibonacci, Lucas, Pell and Companion-Pell sequences and their generalizations as we see in Tables 1 and 2.

In Horadam's notation [9] these are $\left\{F_{k, n}(1, k ; k,-1)\right\},\left\{L_{k, n}(2, k ; k,-1)\right\}$. These are extensions of [10]. The cases $\left\{w_{k, n}(a, b ; 1,-q)\right\}$ are different but similar. Examples of (2.6) and 2.7 include

$$
\begin{array}{ll}
\alpha_{1}^{1}=\frac{1+1 \sqrt{5}}{2}, & \beta_{1}^{1}=\frac{1-1 \sqrt{5}}{2}, \\
\alpha_{1}^{2}=\frac{6+1 \sqrt{5}}{2} & \beta_{1}^{2}=\frac{6-1 \sqrt{5}}{2}, \\
\alpha_{2}^{1}=\frac{2+1 \sqrt{8}}{2} & \beta_{2}^{1}=\frac{2-1 \sqrt{8}}{2}, \\
\alpha_{2}^{2}=\frac{6+2 \sqrt{8}}{2} & \beta_{2}^{2}=\frac{6-2 \sqrt{8}}{2} .
\end{array}
$$

Thus, Equations (2.6), (2.7) and (2.8) generate the traditional Binet formulas for the general terms [11]; that is, 


$$
\alpha_{k}^{n}-\beta_{k}^{n}=P_{k, n} \sqrt{k^{2}+4}
$$

and

$$
\alpha_{k}^{n}+\beta_{k}^{n}=Q_{k, n} .
$$

We see then that as a generalization of (2.5):

$$
\frac{P_{k, n}}{P_{k, n-1}}=\frac{\alpha_{k}^{n}-\beta_{k}^{n}}{\alpha_{k}^{n-1}-\beta_{k}^{n-1}} \rightarrow \alpha_{k}^{1}=\frac{Q_{k, 1}+P_{k, 0} \sqrt{k^{2}+4}}{2} \text { as } n \rightarrow \infty .
$$

For examples, from Tables 2 and 3:

$$
\begin{aligned}
& \frac{P_{1,5}}{P_{1,4}} \approx 1.6000, \frac{P_{1,6}}{P_{1,5}} \approx 1.6250, \ldots, \alpha_{1}^{1}=\frac{1+1 \sqrt{5}}{2} \approx 1.6180 \\
& \frac{P_{2,5}}{P_{2,4}} \approx 2.4137, \frac{P_{2,6}}{P_{2,5}} \approx 2.4142, \ldots, \alpha_{2}^{1}=\frac{2+1 \sqrt{8}}{2} \approx 2.4142 ; \\
& \frac{P_{3,5}}{P_{3,4}} \approx 3.3028, \frac{P_{3,6}}{P_{3,5}} \approx 3.3028, \ldots, \alpha_{3}^{1}=\frac{3+1 \sqrt{13}}{2} \approx 3.3027,
\end{aligned}
$$

and so on.

\begin{tabular}{|c|c|c|c|c|c|c|c|}
\hline $\boldsymbol{k} \downarrow$ & $\mathbf{0}$ & $\mathbf{1}$ & $\mathbf{2}$ & $\mathbf{3}$ & $\mathbf{4}$ & $\mathbf{5}$ & $\mathbf{6}$ \\
\hline $\mathbf{1}$ & 1 & 1 & 2 & 3 & 5 & 8 & 13 \\
\hline $\mathbf{2}$ & 1 & 2 & 5 & 12 & 29 & 70 & 169 \\
\hline $\mathbf{3}$ & 1 & 3 & 10 & 33 & 109 & 360 & 1,189 \\
\hline $\mathbf{4}$ & 1 & 4 & 17 & 72 & 305 & 1,292 & 5,473 \\
\hline $\mathbf{5}$ & 1 & 5 & 26 & 135 & 701 & 3,640 & 17,901 \\
\hline $\mathbf{6}$ & 1 & 6 & 37 & 228 & 1,405 & 8,658 & 53,353 \\
\hline $\mathbf{7}$ & 1 & 7 & 50 & 357 & 2,549 & 18,200 & 129,949 \\
\hline
\end{tabular}

Table 2. Some values of $P_{k, n}$

\begin{tabular}{|c|c|c|c|c|c|c|c|}
\hline $\boldsymbol{k} \downarrow$ & $\mathbf{0}$ & $\mathbf{1}$ & $\mathbf{2}$ & $\mathbf{3}$ & $\mathbf{4}$ & $\mathbf{5}$ & $\mathbf{6}$ \\
\hline $\mathbf{1}$ & 2 & 1 & 3 & 4 & 7 & 11 & 18 \\
\hline $\mathbf{2}$ & 2 & 2 & 6 & 14 & 34 & 82 & 198 \\
\hline $\mathbf{3}$ & 2 & 3 & 11 & 36 & 119 & 393 & 1,298 \\
\hline $\mathbf{4}$ & 2 & 4 & 18 & 76 & 322 & 1,364 & 5,778 \\
\hline $\mathbf{5}$ & 2 & 5 & 27 & 140 & 727 & 3,775 & 19,602 \\
\hline $\mathbf{6}$ & 2 & 6 & 38 & 234 & 1,442 & 8,886 & 54,758 \\
\hline $\mathbf{7}$ & 2 & 7 & 51 & 364 & 2,599 & 18,557 & 132,498 \\
\hline
\end{tabular}

Table 3. Some values of $P_{k, n}$ 


\section{Powers of the coefficient in the recurrence relation}

We can extend the recurrence relation (2.8) by, for example, considering powers of the coefficient.

$$
P_{k, m, n+1}=k^{m} P_{k, m, n}+P_{k, m, n-1}, n \geq 1,
$$

with initial conditions as before, so that, for instance, $\left\{P_{1, m, n}\right\} \equiv\left\{F_{n}\right\},\left\{P_{2,1, n}\right\} \equiv\left\{P_{n}\right\}$, the ordinary Pell numbers. This is an extension of [12]. We shall specifically focus on $\left\{P_{2, m, n}\right\}$ here; that is,

$$
P_{2, m, n+1}=2^{m} P_{2, m, n}+P_{2, m, n-1}, n \geq 1 \text {. }
$$

For instance,

\begin{tabular}{|c|c|c|c|c|c|c|c|}
\hline $\boldsymbol{m} \downarrow$ & $\mathbf{0}$ & $\mathbf{1}$ & $\mathbf{2}$ & $\mathbf{3}$ & $\mathbf{4}$ & $\mathbf{5}$ & $\mathbf{6}$ \\
\hline $\mathbf{1}$ & 1 & 2 & 5 & 12 & 29 & 70 & 169 \\
\hline $\mathbf{2}$ & 1 & 2 & 9 & 38 & 161 & 682 & 2,889 \\
\hline $\mathbf{3}$ & 1 & 2 & 17 & 138 & 1,121 & 9,106 & 73,969 \\
\hline $\mathbf{4}$ & 1 & 2 & 33 & 530 & 8,513 & 136,738 & $2,196,321$ \\
\hline
\end{tabular}

Table 4. Some values of $P_{2, m, n}$

If we wish to extend to an arbitrary order matrix, $r$, for modifications of the van der Laan and Pell-Padovan sequences [13] we can use matrices

$$
P(m)_{r \times r}=\left[\begin{array}{cccc}
P_{k, m, n} & P_{k, m, n+1} & \ldots & P_{k, m, n+r-1} \\
P_{k, m, n-1} & P_{k, m, n} & \ldots & P_{k, m, n+r-2} \\
& & \ldots & \\
P_{k, m, n-r+1} & P_{k, m, n-r+2} & \ldots & P_{k, m, n}
\end{array}\right]
$$

and

$$
R(m)_{r \times r}=\left[\begin{array}{cccc}
P_{k, m, n} & P_{k, m+1, n} & \ldots & P_{k, m+r-1, n} \\
P_{k, m-1, n} & P_{k, m, n} & \ldots & P_{k, m+r-2, n} \\
& & \ldots & \\
P_{k, m-r+1, n} & P_{k, m-r+2, n} & \ldots & P_{k, m, n}
\end{array}\right]
$$

with a corresponding

$$
Q(m)_{r \times r}=\left[\begin{array}{ccccc}
2^{m} & 0 & \ldots & 0 & -1 \\
1 & 0 & \ldots & 0 & 0 \\
& & \ldots & & \\
0 & 0 & \ldots & 1 & 0
\end{array}\right]
$$

so that

$$
Q(m)_{2 \times 2}=\left[\begin{array}{cc}
2^{m} & -1 \\
1 & 0
\end{array}\right]
$$


and

$$
P(m)_{2 \times 2}=\left[\begin{array}{cc}
P_{2, m, n} & P_{2, m, n+1} \\
P_{2, m, n-1} & P_{2, m, n}
\end{array}\right] .
$$

The last two matrices can obviously be used to generate properties analogous to well-known Fibonacci sequence properties. It would also seem that for analogues of the Simson identity:

$$
\begin{aligned}
& \operatorname{det} P(1)_{2 \times 2}=(-1)^{n} 1 \\
& \operatorname{det} P(2)_{2 \times 2}=(-1)^{n} 5 \\
& \operatorname{det} P(3)_{2 \times 2}=(-1)^{n} 13 \\
& \operatorname{det} P(4)_{2 \times 2}=(-1)^{n} 29
\end{aligned}
$$

so that

$$
\operatorname{det} P(m)_{2 \times 2}=(-1)^{n} w_{m}
$$

in which

$$
w_{m}=w_{m-1}+2^{m}, n \geq 0, w_{1}=1 .
$$

Equation (3.5) is a first order non-homogeneous difference equation with a standard solution of

$$
w_{m}=2^{m+1}-3 .
$$

We note that (3.3) confirms Simson's identity, but (3.4) and following show that it is part of a bigger picture. Arbitrary order extensions can also be defined by

$$
S_{k, m, n+1}=k^{m} S_{k, m, n}+S_{k, m, n-r+1}, n \geq 1,
$$

with initial conditions $S_{k, m, n}=n+1, n=0,1,2, \ldots, r-1$. For instance, when $r=3$ and $k=2$, we get the sequences set out in Table 5:

\begin{tabular}{|c|c|c|c|c|c|c|c|}
\hline $\boldsymbol{m} \downarrow$ & $\mathbf{0}$ & $\mathbf{1}$ & $\mathbf{2}$ & $\mathbf{3}$ & $\mathbf{4}$ & $\mathbf{5}$ & $\mathbf{6}$ \\
\hline $\mathbf{1}$ & 1 & 2 & 3 & 7 & 16 & 35 & 73 \\
\hline $\mathbf{2}$ & 1 & 2 & 3 & 13 & 54 & 219 & 889 \\
\hline $\mathbf{3}$ & 1 & 2 & 3 & 25 & 202 & 1,619 & 12,977 \\
\hline $\mathbf{4}$ & 1 & 2 & 3 & 49 & 786 & 12,579 & 201,313 \\
\hline
\end{tabular}

Table 5. Some values of $S_{2, m, n}$

From this we can see that third order analogues of the Simson identity have the formats

$$
\begin{aligned}
& \operatorname{det} P(1)_{3 \times 3}=(-1)^{n} 8 \\
& \operatorname{det} P(2)_{3 \times 3}=(-1)^{n} 94 \\
& \operatorname{det} P(3)_{3 \times 3}=(-1)^{n} 554 \\
& \operatorname{det} P(4)_{3 \times 3}=(-1)^{n} 2626 .
\end{aligned}
$$




\section{Concluding comments}

It can be readily confirmed that there are many elegant analogues of well-known Fibonacci and Lucas numbers. For instance, (allowing for the notational variations in the initial values

$$
L_{k, n}=F_{k, n}+F_{k, n-2}
$$

and

$$
(-1)^{n+1}=F_{k, n-1} F_{k, n+1}-F_{k, n}^{2}
$$

as a variation of Simson's identity. Somewhat similar ideas for extensions may be found in [14].

It is also of interest to explore some of the properties of Tables 2 and 3. For example, Equation (2.8) is a partial difference equation with $n$ as the variable, and we have so far considered the row sequences of these tables. If however we make $k$ the variable (that is, consider the column sequences), then it can be readily confirmed by calculation from Table 2 that for $n=0,1,2$, it holds that

$$
\begin{aligned}
F_{k, 0} & =F_{k-1,0} \\
F_{k, 1} & =F_{k-1,1}+1 \\
F_{k, 2} & =F_{k-1,2}+F_{k, 1}+F_{k-1,1} \\
& =F_{k-1,2}+2 F_{k-1,1}+1
\end{aligned}
$$

but is there a partial difference equation in $k$ (or $m$ ) for all $n$ ?

It should be noted that the Fibonacci sequence equation for the Golden Ratio Family, $\left\{\varphi_{n}\right\}$ $[15]$ is

$$
F_{n+1}=F_{n}+r F_{n-1},
$$

with $r$ a structural variable from Modular Ring Theory, contrasts with Equation (2.8) where $k$ is linked to the Pell sequences and $P_{k, n}$ is the multiplied component. The original Golden Ratio Family surd is

$$
\varphi_{a}=\frac{1+\sqrt{a}}{2}
$$

with $(a=4 r+1) \in \overline{1}_{4} \subset Z_{4}$, a modular ring, in contrast with the surds constructed here with types of generalized Pell numbers. Since $k^{2}+4=k^{2}+2^{2}$, when $k$ is odd the sum of squares must equal $4 r+1$ or $a$. This provides a link between the two golden ratio families, and also with primitive Pythagorean triples [16].

\section{References}

[1] Livio, M. (2002) The Golden Ratio. Golden Books, New York.

[2] Atanassov, K., Atanassova, V., Shannon, A., \& Turner, J. (2002) New Visual Perspectives on the Fibonacci Numbers. World Scientific, New York.

[3] Hoggatt, V. E., Jr. (1969) Fibonacci and Lucas Numbers. Houghton-Mifflin, Boston. 
[4] Tee, G. J. (2003) Russian Peasant Multiplication and Egyptian Division in Zeckendorf Arithmetic. Australian Mathematical Society Gazette, 30, 267-276.

[5] Stakhov, O. (1997) Computer Arithmetic based on Fibonacci Numbers and Golden Section: New Information and Arithmetic Computer Foundations. Vinnitsa: Ukrainian Academy of Engineering Sciences, Ch.2.

[6] Graham, R. L., Knuth, D. E., \& Patashnik, O. (1992) Concrete Mathematics: A Foundation for Computer Science. Addison-Wesley, Reading, MA.

[7] Shannon, A. G., \& Leyendekkers, J. V. (2015) The Golden Ratio family and the Binet equation. Notes on Number Theory and Discrete Mathematics, 21(2), 35-42.

[8] Falcon, S. (2018) Some new formulas on the K-Fibonacci numbers. Journal of Advances in Mathematics, 14(1), 7439-7445.

[9] Horadam, A. F. (1965) Basic properties of a certain generalized sequence of numbers. The Fibonacci Quarterly, 3(3), 161-176.

[10] Cook, C. K., \& Shannon, A. G. (2006) Generalized Fibonacci and Lucas sequences with Pascal-type arrays. Notes on Number Theory and Discrete Mathematics, 12(4), 1-9.

[11] Shannon, A. G., Anderson, P. G., \& Horadam, A. F. (2006) Properties of Cordonnier, Perrin and van der Laan Numbers. International Journal of Mathematical Education in Science and Technology, 37(7), 825-831.

[12] Shannon, A. G., \& Horadam, A. F. (2004) Generalized Pell numbers and polynomials. In: Fredric T. Howard (ed.) Applications of Fibonacci Numbers, Volume 9. Dordrecht/Boston/ London: Kluwer, 213-224.

[13] Deveci, Ö., \& Shannon, A. G. (2017) Pell-Padovan-circulant sequences and their applications. Notes on Number Theory and Discrete Mathematics, 23(3), 100-114.

[14] Shannon, A. G., \& Leyendekkers, J. V. (2018) The Fibonacci Numbers and Integer Structure. Nova Science Publishers, New York.

[15] Leyendekkers, J. V., \& Shannon, A. G. (2015) The Golden Ratio Family and generalized Fibonacci Numbers. Journal of Advances in Mathematics, 10(1), 3130-3137.

[16] Leyendekkers, J. V., \& Rybak, J. M. (1995) Pellian sequences derived from Pythagorean triples. International Journal of Mathematical Education in Science and Technology, 26(6), 903-922. 